\title{
Retrieving qubit information despite decoherence
}

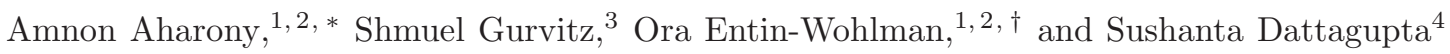 \\ ${ }^{1}$ Department of Physics and the Ilse Katz Center for Meso- and Nano-Scale Science and Technology, \\ Ben-Gurion University, Beer Sheva 84105, Israel \\ ${ }^{2}$ Albert Einstein Minerva Center for Theoretical Physics, \\ Weizmann Institute of Science, Rehovot 76100, Israel \\ ${ }^{3}$ Department of Particle Physics and Astrophysics, \\ Weizmann Institute of Science, Rehovot 76100, Israel \\ 4 Indian Institute of Science Education and Research-Kolkata, Mohanpur 741252, India
}

(Dated: July 26, 2021)

\begin{abstract}
The time evolution of a qubit, consisting of two single-level quantum dots, is studied in the presence of telegraph noise. The dots are connected by two tunneling paths, with an AharonovBohm flux enclosed between them. Under special symmetry conditions, which can be achieved by tuning gate voltages, there develops partial decoherence: at long times, the off-diagonal element of the reduced density matrix (in the basis of the two dot states) approaches a non-zero value, generating a circulating current around the loop. The flux dependence of this current contains full information on the initial quantum state of the qubit, even at infinite time. Small deviations from this symmetry yield a very slow exponential decay towards the fully-decoherent limit. However, the amplitudes of this decay also contain the full information on the initial qubit state, measurable either via the current or via the occupations of the qubit dots.
\end{abstract}

PACS numbers: 03.67.-a, 05.40.-a, 03.65.Yz, 03.67.Pp

\section{INTRODUCTION AND SUMMARY}

Quantum computation operates on information stored in "qubits", which are superpositions of two basic quantum states, 1

$$
\left|\psi_{0}\right\rangle=\cos \alpha|1\rangle+e^{i \gamma} \sin \alpha|2\rangle,
$$

with two real parameters $\alpha$ and $\gamma$. In one realization of a solid-state qubit, the two basic states represent singlelevel quantum dots, $\stackrel{2}{=}$ where the superposition state (11), representing a single electron on the two dots, may be given as input, or modified by tuning the dot energies $\epsilon_{1,2}$ and the inter-dot tunneling $J_{12}$. In a tight-binding language, the Hamiltonian of the qubit is given by

$$
\mathcal{H}_{\mathrm{q}}=\epsilon_{1} a_{1}^{\dagger} a_{1}+\epsilon_{2} a_{2}^{\dagger} a_{2}-\left(J_{12} a_{1}^{\dagger} a_{2}+\text { h.c. }\right)
$$

where $a_{n}^{\dagger}$ creates an electron on $\operatorname{dot} n, a_{n}^{\dagger}|0\rangle \equiv|n\rangle$. The average energy $\epsilon \equiv\left(\epsilon_{1}+\epsilon_{2}\right) / 2$ does not affect the dynamics of the qubit, $\stackrel{3}{=}$ and therefore we set it equal to zero. The dynamics is then determined by the energy gap $\Delta=\epsilon_{1}-\epsilon_{2}$ and by $J_{12}$,

$$
\mathcal{H}_{\mathrm{q}}=(\Delta / 2)\left(a_{1}^{\dagger} a_{1}-a_{2}^{\dagger} a_{2}\right)-\left(J_{12} a_{1}^{\dagger} a_{2}+\text { h.c. }\right) .
$$

In the literature on $\mathrm{NMR} \stackrel{4-6}{\underline{4}} \mathcal{H}_{\mathrm{q}}$ is often written in the equivalent pseudo-spin form

$$
\mathcal{H}_{\mathrm{q}}=\mathbf{B} \cdot \boldsymbol{\sigma}
$$

where $\boldsymbol{\sigma}$ represents the three Pauli matrices and where $B_{z}=\Delta / 2, B_{x}-i B_{y}=-J_{12}$. Another physical realization of a qubit involves a superconducting Josephson junction. $\frac{7}{}$
Clearly, quantum computation requires the stability of the quantum state stored on each qubit, and therefore it can be used only while this state remains coherent $\frac{8}{8}$ Interactions between qubits and their environment, including input-output measurement devices, can cause decoherence which destroys the information stored in the qubits. Therefore, it is important to study the time evolution of the qubit state in the presence of the environment. To concentrate on the state of the qubit, one traces over the states of the environment, ending up with the $2 \times 2$ reduced density matrix of the qubit itself, $\rho(t) \equiv \operatorname{Tr}_{\text {env }}[|\Psi(t)\rangle\langle\Psi(t)|]$, where $|\Psi\rangle$ is the combined state of the qubit and the environment. In many cases, the coupling to the environment yields full asymptotic decoherence, for which the elements of reduced density matrix approach the fully-mixed state,

$$
\rho_{n m}(t \rightarrow \infty)=\delta_{n m} / 2
$$

independent of which basis is used for the Hilbert space. In these cases, the information on the initial qubit quantum state is totally lost. However, in some symmetric cases there exist decoherence-free subspaces, which decouple from the environment, so that at least some of the information on the initial quantum state remains protected $, 9,10$

Here we discuss special cases in which the full information on the initial qubit state can be retrieved, even after a long time, despite decoherence. Since our results rely mainly on symmetry, we expect them to hold whenever the required symmetry holds, irrespective of the specific nature of the environment and its coupling to the qubit. To demonstrate our point we consider the simplest model for decoherence, where the environment generates a single parameter which fluctuates randomly between 
two values, as in telegraph noise 11 We thus replace the Hamiltonian of the qubit by $\mathcal{H}_{\mathrm{q}} \rightarrow \mathcal{H}_{\mathrm{q}}+f(t) \mathcal{V}$, where $f(t)$ jumps stochastically between +1 and -1 . Indeed, such jumps in $f(t)$ may arise e.g. due to equilibrium or non-equilibrium sources of noise, e.g. from background (natural) charge fluctuations $\underline{12.13}$ or to a capacitive coupling to a current which flows through a (tunable) neighboring single-electron transistor $\stackrel{14.15}{=}$

In principle, the Hamiltonian of the coupling between the qubit and the noise source, $\mathcal{V}$, may involve the same operators which appear in Eq. (3):

$$
\mathcal{V}=\left(\zeta_{\Delta} / 2\right)\left(a_{1}^{\dagger} a_{1}-a_{2}^{\dagger} a_{2}\right)-\left(\zeta_{J} a_{1}^{\dagger} a_{2}+\text { h.c. }\right),
$$

where the $\zeta$ 's are fixed coefficients, whose size measures the coupling between the qubit and the environment. In the special cases which we discuss, $\mathcal{V}$ commutes with $\mathcal{H}_{\mathrm{q}}$. This requires specific ratios between the coefficients in Eq. (6) and those in Eq. (3). Such ratios can be achieved experimentally by tuning gate voltages which control the coefficients in Eq. (3), or by a careful placing of the source of the noise relative to the qubit. When these conditions are obeyed, one can switch to a basis which diagonalizes $\mathcal{H}_{\mathrm{q}}$. In this basis, the diagonal elements of the reduced density matrix are independent of time, while the off-diagonal elements decay to zero, reflecting pure dephasing. Translated to the dot basis of the Hilbert space, this implies that

$$
\begin{aligned}
& \operatorname{Tr}_{\text {env }}\left[\left\langle\Psi(t)\left|\mathcal{H}_{\mathrm{q}}\right| \Psi(t)\right\rangle\right] \\
& \equiv \frac{\Delta}{2}\left[\rho_{11}(t)-\rho_{22}(t)\right]-2 \operatorname{Re}\left[J_{12} \rho_{21}(t)\right]=\text { const. }
\end{aligned}
$$

independent of time. Thus, the density matrix never reaches the fully-mixed state (5). We refer to this situation as partial decoherence.

The decoherence of qubits due to telegraph noise has been treated in several earlier papers $\underline{12-16}$ In a situation where both $\Delta$ and $J_{12}$ are present, and both are noisy, the qubit's reduced density matrix usually decays exponentially towards the fully-mixed state $\frac{17}{}$ Alternatively, Itakura and Tokura $\frac{12}{2}$ considered the special case without a gap between the dot energies, $\Delta=\zeta_{\Delta}=0$ [cf. Eqs. (31) and (6)], and found that when $J_{12}$ and $\zeta_{J}$ are real, then both $\mathcal{H}_{\mathrm{q}}$ and $\mathcal{V}$ are symmetric under the interchange $1 \leftrightarrow 2$, and therefore the 'bonding' and 'anti-bonding' symmetric and antisymmetric states $| \pm\rangle=(|1\rangle \pm|2\rangle) / \sqrt{2}$ are eigenstates of both. In that case, the off-diagonal element $\rho_{+-}$oscillates and decays to zero with the 'dephasing time' $T_{2}$. Below we show that this example is a special case of a broad family of systems, all of which exhibit partial decoherence [cf. Eq. (77]].

The procedures proposed below require that $J_{12}$ should be complex and tunable experimentally. To achieve this, we connect the two qubit quantum dots via two separate tunneling channels, with energies $J_{u}$ and $J_{d}$ (Fig. 1), while a magnetic flux $\Phi$ is enclosed between them. Utilizing gauge invariance, the combined tunneling coupling
$J_{12}$ becomes

$$
J_{12}=J_{u}+J_{d} e^{i \phi} \equiv\left|J_{12}\right| e^{i \theta},
$$

with the Aharonov-Bohm phase $\phi=2 \pi \Phi / \Phi_{0}$, where $\Phi_{0}=h c / e$ is the flux unit. Both $J_{u}$ and $J_{d}$ (which are chosen real) can be tuned via gate voltages, and the phase $\theta$ can be tuned via the magnetic flux.

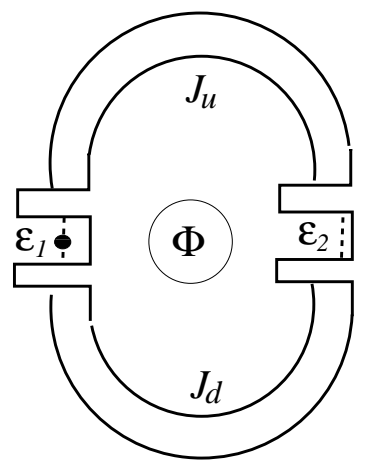

FIG. 1: The two-dot qubit with tunneling channels $u$ and $d$.

To demonstrate the result (7) we present what we term 'the symmetric case', in which there is no energy gap, $\Delta=\zeta_{\Delta}=0$, but $J_{12}$ can still be complex. The vanishing of $\zeta_{\Delta}$ is achieved when the source of noise is located symmetrically relative to the two dots, or when the correlation length of the noise is larger than the qubit's size. For an arbitrary flux $\Phi$, and for given values of the noise coefficients $\zeta_{u}$ and $\zeta_{d}$ [defined via $J_{u, d} \rightarrow J_{u, d}+f(t) \zeta_{u, d}$ ], we show that an appropriate tuning of either $J_{u}$ or $J_{d}$ (via corresponding gate voltages) suffices to bring the system into a special symmetric case, in which its reduced density matrix never reaches the fully-mixed state (5). Instead, at $t \rightarrow \infty$ it approaches the asymptotic limit

$$
\rho \rightarrow\left(\begin{array}{cc}
1 / 2 & e^{i \theta} \operatorname{Re}\left[e^{-i \theta} \rho_{12}(0)\right] \\
e^{-i \theta} \operatorname{Re}\left[e^{-i \theta} \rho_{12}(0)\right] & 1 / 2
\end{array}\right),
$$

where $\rho_{12}(0)=\left\langle 1 \mid \psi_{0}\right\rangle\left\langle\psi_{0} \mid 2\right\rangle=e^{-i \gamma} \sin (2 \alpha) / 2$ [see Eq. (11)] and where $\theta$ is the phase of the complex $J_{12}$, see Eq. (8). Equation (9) is a special case of Eq. (7), for $\Delta=0$.

The non-zero complex off-diagonal element $\rho_{12}$ generates a circulating current around the loop, which can be used to retrieve the qubit's information. The equation of motion for the number operator $a_{1}^{\dagger} a_{1}$ is

$$
\partial_{t} a_{1}^{\dagger} a_{1}=\hat{\mathcal{I}}_{u}+\hat{\mathcal{I}}_{d},
$$

where the operator $\hat{\mathcal{I}}_{u}$ and $\hat{\mathcal{I}}_{d}$ represent the currents into dot 1 via the channels $u$ and $d$, respectively:

$$
\hat{\mathcal{I}}_{u}=i\left[J_{u} a_{1}^{\dagger} a_{2}-\text { h.c. }\right], \quad \hat{\mathcal{I}}_{d}=i\left[J_{d} e^{i \phi} a_{1}^{\dagger} a_{2}-\text { h.c. }\right] .
$$

Therefore, the net current into site 1 is

$$
\mathcal{I}=\left\langle\Psi\left|\hat{\mathcal{I}}_{u}-\hat{\mathcal{I}}_{d}\right| \Psi\right\rangle=\left\langle\Psi\left|2 \hat{\mathcal{I}}_{u}-\partial_{t} a_{1}^{\dagger} a_{1}\right| \Psi\right\rangle .
$$


In the asymptotic stationary limit we can drop the last term. Tracing over the environment then yields the conditional average $\left\langle\Psi(t)\left|J_{u} a_{1}^{\dagger} a_{2}\right| \Psi(t)\right\rangle \rightarrow J_{u} \rho_{21}$. For the symmetric case, we find below that averaging over the noise yields

$$
\mathcal{I} \rightarrow 2 J_{0} \operatorname{Im}\left[\rho_{12}(\infty)\right]=J_{0} \sin (2 \alpha) \sin \theta \cos (\theta+\gamma),
$$

where $J_{0}$ is the average of $J_{u}+\zeta_{u} f(t)$ over the noise. As expected, this current vanishes when $\phi$ is an integer multiple of $\pi$ [when also $\theta=0$, see Eq. (8)] ]. However, at nontrivial fluxes $\mathcal{I}$ is non-zero, despite decoherence. This current generates an orbital magnetic moment of the electron circulating the loop. Measuring its $\phi$-dependence can yield both $\alpha$ and $\gamma$ [see Eq. (11)], namely the full information stored initially! Unlike the usual equilibrium persistent current, which is an odd function of the flux (as required by time-reversal symmetry) $\frac{18}{2}$ the current here is neither odd nor even in the flux. This peculiar flux dependence apparently results from the averaging over the noise, which breaks time-reversal symmetry.

Following our analysis of the symmetric case, we also consider small deviations from this symmetry, and find that such deviations lead asymptotically to the fullymixed state, as also found e. g. in Refs. 1214 and in references given there. However, for small deviations from symmetry there is a distinct separation of time scales. After a transient oscillatory stage, the elements of the density matrix develop a very slow simple exponential decay towards the asymptotic fully-mixed state. These slowly decaying terms also include a non-zero difference in the dot occupations, $z=\rho_{11}-\rho_{22}$ (which approached zero relatively quickly in the symmetric case). This difference can also be measured experimentally. We show that the amplitudes of these exponential terms also contain the full information on the initial state of the qubit. This information can therefore be extracted even after the transient stage.

The plan of the paper is as follows. The formalism for the telegraph noise is reviewed in Sec. II. Section III then presents several physical environments which can generate telegraph noise of the kind discussed here. The general conditions for partial decoherence, and the example of the symmetric case, are presented in Sec. IV. In Sec. V we then introduce deviations from symmetry, and Sec. VI contains a discussion of our results.

\section{TELEGRAPH NOISE}

A treatment of the equation of motion with the underlying stochasticity in $f(t)$ can be found in the literature on the theory of lineshapes $\stackrel{4,19}{=}$ Here we follow Blume, $\stackrel{20}{,}$ and average the density matrix $\rho(t)$ over the histories of the stochastic noise, under the condition that at time $t$ the random function $f(t)$ has the values $b=1$ or -1 . We then define a 2 -component vector (denoted by bold letters) $\boldsymbol{\rho}(t)$, such that its $b$-th component represents this conditional average $\rho(t, b)$. At the end one may average over the stochastic process,

$$
\rho(t)=\sum_{b= \pm 1} \rho(t, b) .
$$

The function $f(t)$ follows a Markov process: 21 it jumps randomly from 1 to -1 (or from -1 to 1 ) with the rate $w_{-+}$(or $\left.w_{+-}\right)$. These jumps in $f(t)$ result from a contact with some noise source. The noise distribution is characterized by the probabilities $p_{ \pm}$to find $f(t)$ at the values \pm 1 . Detailed balance then implies the relation $p_{-} w_{+-}=p_{+} w_{-+}$, and therefore the jump rates can be written as

$$
w_{ \pm \mp}=\lambda p_{ \pm},
$$

where $\lambda=w_{+-}+w_{-+}$represents the inverse time associated with the noise.

Our main purpose here is to calculate the time evolution of $\boldsymbol{\rho}$. The equations of motion for the conditional averages $\rho(t, b)$ are

$$
\begin{aligned}
\partial_{t} \rho(t, b) & =-i\left[\mathcal{H}_{\mathrm{q}}+b \mathcal{V}, \rho(t, b)\right] \\
& +w_{b,-b} \rho(t,-b)-w_{-b, b} \rho(t, b),
\end{aligned}
$$

where we use $\hbar=1$ throughout. The first term on the right-hand side applies if $f(t)$ remains unchanged at time $t$ (i.e. stays equal to $b$ ). In this case, the time evolution of the density matrix proceeds with the Liouville operator which corresponds to the original Hamiltonian, with $f(t)=b$. The last two terms arise if $f(t)$ flips exactly at time $t$, either from $-b$ to $b$ (second term) or from $b$ to $-b$ (last term).

Each element of the $2 \times 2$ reduced density matrix now becomes a $2-$ component vector, $\boldsymbol{\rho}_{n m}$, and Eq. (16) can be written in matrix form,

$$
i\left(\mathbf{I} \partial_{t}-\mathbf{W}\right) \boldsymbol{\rho}_{n m}=\boldsymbol{\Delta}_{n m} \boldsymbol{\rho}_{n m}-\sum_{\ell}\left(\mathbf{J}_{n \ell} \boldsymbol{\rho}_{\ell m}-\boldsymbol{\rho}_{n \ell} \mathbf{J}_{\ell m}\right),
$$

with $n, m=1,2$. Here, each parameter in the Hamiltonian $\mathcal{H}_{\mathrm{q}}+b \mathcal{V}$ is replaced by a diagonal $2 \times 2$ matrix. For our specific two-dot system, $\boldsymbol{\Delta}_{n m}=\mathbf{J}_{n m}=0$ for $n=m$ while $\boldsymbol{\Delta}_{12} \equiv \boldsymbol{\Delta} \equiv \Delta \mathbf{I}+\zeta_{\Delta} \boldsymbol{\sigma}_{z}$ represents the energy gap variable and $J_{12} \rightarrow \mathbf{J}_{12} \equiv J_{12} \mathbf{I}+\zeta_{J} \boldsymbol{\sigma}_{z}$ represents the hopping matrix element ( $\mathbf{I}$ is the $2 \times 2$ unit matrix). The relaxation matrix $\mathbf{W}$ consists of the stochastic hopping probabilities of the noise,

$$
\mathbf{W}=\left(\begin{array}{cc}
-w_{-+} & w_{+-} \\
w_{-+} & -w_{+-}
\end{array}\right) \equiv \lambda(\mathbf{T}-\mathbf{I})
$$

[see Eq. (15)], where

$$
\mathbf{T} \equiv\left(\begin{array}{ll}
p_{+} & p_{+} \\
p_{-} & p_{-}
\end{array}\right) \equiv\left[\mathbf{I}+\boldsymbol{\sigma}_{x}+\Delta p\left(\boldsymbol{\sigma}_{z}+i \boldsymbol{\sigma}_{y}\right)\right] / 2
$$

and $\Delta p \equiv p_{+}-p_{-}$. 
The matrix $\mathbf{W}$ determines the time evolution of the matrix $\mathbf{P}(t)$, where $(b|\mathbf{P}(t)| a)$ is the probability for the stochastic variable to start at $t=0$ with the value $a$ and end at $t>0$ with the value $b: \mathbf{P}$ obeys the equation $\partial_{t} \mathbf{P}(t)=\mathbf{W} \mathbf{P}(t)$, and therefore

$$
\mathbf{P}(t)=e^{\mathbf{W} t}=\mathbf{T}+(\mathbf{I}-\mathbf{T}) e^{-\lambda t},
$$

where we have used the identity $\mathbf{T}^{2}=\mathbf{T}$. At infinite time, $\mathbf{P}$ approaches $\mathbf{T}$, and thus $(b|\mathbf{P}(\infty)| a)=p_{b}(a, b=$ \pm ), independent of the initial value $a$. Below we use $\mathbf{P}(t)$ for some of the solutions for the density matrix. It is customary to characterize the noise by its spectral function, defined via

$$
S(\omega)=2 \operatorname{Re} \int_{0}^{\infty} \sum_{b, b^{\prime}} p_{b^{\prime}}(b-\bar{b})\left(b|\mathbf{P}(t)| b^{\prime}\right)\left(b^{\prime}-\bar{b}\right) e^{i \omega t} d t,
$$

where $\bar{b}=\sum_{b} p_{b} b=\Delta p$. Using Eq. (20) one finds

$$
S(\omega)=\frac{8 w_{+-} w_{-+}}{\lambda\left(\omega^{2}+\lambda^{2}\right)} \equiv \frac{8 \lambda p_{+} p_{-}}{\omega^{2}+\lambda^{2}} .
$$

Below we relate various decay times with special values of $S(\omega)$.

A convenient way to solve Eqs. (17) is by employing the Laplace transform,

$$
\widetilde{\boldsymbol{\rho}}(s)=\int_{0}^{\infty} d t e^{-s t} \boldsymbol{\rho}(t) .
$$

The equations of motion (17) then become

$$
\begin{array}{r}
(\mathbf{I} s-\mathbf{W}) \widetilde{\boldsymbol{\rho}}_{n m}=\boldsymbol{\rho}_{n m}(0)-i \boldsymbol{\Delta}_{n m} \widetilde{\boldsymbol{\rho}}_{n m} \\
+i \sum_{\ell}\left(\mathbf{J}_{n \ell} \widetilde{\boldsymbol{\rho}}_{\ell m}-\widetilde{\boldsymbol{\rho}}_{n \ell} \mathbf{J}_{\ell m}\right) .
\end{array}
$$

Since the initial values of the density matrix, $\rho_{n m}(0) \equiv$ $\left\langle n \mid \psi_{0}\right\rangle\left\langle\psi_{0} \mid m\right\rangle$, do not depend on the stochastic noise, we assume the latter to be in its steady state, and identify the initial vector $\boldsymbol{\rho}_{n m}(0)$ with

$$
\boldsymbol{\rho}_{n m}(0) \equiv \rho_{n m}(0) \mathbf{p}_{0}, \quad \mathbf{p}_{0} \equiv\left(\begin{array}{c}
p_{+} \\
p_{-}
\end{array}\right) .
$$

Note that $\mathbf{p}_{0}$ is an eigenstate of $\mathbf{T}$, with eigenvalue 1 , and therefore $\mathbf{P}(t) \mathbf{p}_{0}=\mathbf{p}_{0}$, so that $\mathbf{p}_{0}$ corresponds to the steady state of the stochastic noise.

One major issue in this paper concerns the asymptotic limit of the density matrix, at long times. Without noise, the eigenvalues of $\mathcal{H}_{\mathrm{q}}$ are $\pm \Omega / 2$, with eigenstates $| \pm\rangle$. In the basis of these eigenstates, $\rho_{ \pm \pm}$remain constant in time, while $\rho_{+-}(t)=e^{-i \Omega t} \rho_{+-}(0)$, with the Rabi frequency

$$
\Omega=\sqrt{\Delta^{2}+4\left|J_{12}\right|^{2}} .
$$

In the presence of noise, the density matrix often approaches a stationary state, so that $\partial_{t} \boldsymbol{\rho}=0$. This is indeed the case in our analysis. One can then find the stationary state by solving the homogeneous linear set of equations (17) in $\boldsymbol{\rho}_{n m}$. Alternatively, one can use Eqs. (24), together with the identity

$$
\lim _{t \rightarrow \infty} \boldsymbol{\rho}(t)=\lim _{s \rightarrow 0} s \widetilde{\boldsymbol{\rho}}(s) .
$$

\section{PHYSICAL MODELS YIELDING TELEGRAPH NOISE}

The reduced density matrix is obtained by solving the equations of motion for the joint density matrix of the qubit and the environment, and then tracing over the environment degrees of freedom. This procedure is quite complicated when the time-dependence of the environment degrees of freedom is influenced by those of the qubit. This influence is called 'back action'. Neglecting this back action implies that one can calculate the time dependence of the environment degrees of freedom separately, independent of the qubit states. In the simplest model discussed here, the qubit couples to the environment only via one degree of freedom, which is represented by its time dependent value $f(t)$. Here we review three examples of models which have been treated in the literature.

Itakura and Tokura 12 already reviewed the literature on background charge fluctuations (see also Refs. 13 and 16). In that case, a single impurity near the qubit is either occupied by an electron or empty, with probabilities $p_{+}$and $p_{-}$and with hopping rates given by Eq. (15), in which $\lambda$ is proportional to $e^{-\Delta E / k_{B} T}$ and $\Delta E$ is the activation energy of the impurity (we asume a large Coulomb blockade, preventing double occupancy). Neglecting the back action of the qubit onto the impurity, this model reduces to the classical telegraph noise one, in which the impurity-qubit coupling generates different coefficients in the intra-qubit Hamiltonian for each state of the impurity.

Another possible model concerns a two-level system with an energy gap $\Delta E$ (e.g. a double potential well

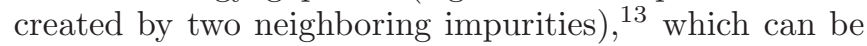
represented by a pseudo-spin $1 / 2$. At equilibrium with a heat bath at temperature $T$, the occupation probabilities obey a Boltzmann distribution $p_{+}=1-p_{-}=$ $\left[1+e^{-\Delta E / k_{B} T}\right]^{-1}$. Each state of this pseudo-spin generates different values for the coupling parameters within the qubit Hamiltonian, again yielding the telegraph noise picture.

It is usually not easy to justify the neglect of the back actions in the above two examples. However, as argued by Galperin et al., 22 back action may be ignored when the dynamics of the fluctuating background charge or the two-level system is governed by its coupling to a thermalizing heat bath, which is much stronger than its coupling to the qubit. The telegraph noise model is also justified in the limit of a very high temperature of this heat bath $\underline{16}$ 
Here we concentrate on yet another example, in which a current between a left and a right reservoirs $L$ and $R$ respectively (held at chemical potentials $\mu_{L}>\mu_{R}$ ) flows through a single electron transistor (SET), located near the qubit. Unlike the above two examples, here the fluctuator is not at equilibrium. The states of the environment (SET plus reservoirs) include states in which an arbitrary number of electrons have moved between the two reservoirs, while the SET can be (singly) occupied or empty. The quantum equations of motion for the density matrix of the combined qubit-SET system were analyzed in Refs. 14 and 15. When the bias voltage $\mu_{L}-\mu_{R}$ is much larger than any other energy in the problem, these authors traced over the environment states, and obtained equations of motion for the reduced qubit density matrix, which are equivalent to our Eqs. (16). In these equations, the rate of an electron entering the SET from the left reservoir, $\Gamma_{L}$, was identified with $w_{+-}$, and the rate of an electron leaving the SET to the right, $\Gamma_{R}$, was identified with $w_{-+}$. Here, $\Gamma_{L}\left(\Gamma_{R}\right.$ is the partial width of the SET level, caused by its coupling to the $L(R)$ reservoir.

When the SET is placed near the qubit, the electron on the latter feels an additional Coulomb potential generated whenever the SET dot is occupied. Denoting the creation operator of an electron on the SET by $c_{0}^{\dagger}$, the coupling between the SET and the qubit is given by

$$
\mathcal{H}_{\text {int }}=c_{0}^{\dagger} c_{0}\left(U_{1} a_{1}^{\dagger} a_{1}+U_{2} a_{2}^{\dagger} a_{2}-\left[U_{J} a_{1}^{\dagger} a_{2}+\text { h.c. }\right]\right) .
$$

The energy $U_{J}$ represents a sum of two matrix elements, associated with the effect of the SET on the hopping between the qubit dots. Assuming the geometry of Fig. 1, and using the same gauge choice as in Eq. (8), these matrix elements can be written as

$$
U_{J}=U_{u}+U_{d} e^{i \phi}
$$

with real $U_{u}$ and $U_{d}$. Using the conclusions of Refs. 14 and 15, one may replace $c_{0}^{\dagger} c_{0}$ in Eq. (28) by a c-number, $[1+f(t)] / 2$. Absorbing the time-independent part in $\mathcal{H}_{\mathrm{q}}$ then yields

$$
\zeta_{\Delta}=\left(U_{1}-U_{2}\right) / 2, \quad \zeta_{u, d}=U_{u, d} / 2
$$

These parameters clearly depend on the relative location of the SET with respect the two dots and the two tunneling paths.

\section{PARTIAL DECOHERENCE}

\section{A. General conditions for partial decoherence}

Here we show that a system develops partial decoherence, i.e. does not approach the fully-mixed state (5), whenever $\mathcal{H}_{\mathrm{q}}$ and $\mathcal{V}$ [Eqs. (3) and [6)] commute with each other, and discuss the conditions for this to happen. The commutator of these operators is given by

$$
\begin{aligned}
{\left[\mathcal{H}_{\mathrm{q}}, \mathcal{V}\right] } & =\left[\left(\Delta \zeta_{J}^{*}-J_{12}^{*} \zeta_{\Delta}\right) a_{1}^{\dagger} a_{2}-\text { h.c. }\right] \\
& +\left(J_{12} \zeta_{J}^{*}-J_{12}^{*} \zeta_{J}\right)\left(a_{1}^{\dagger} a_{1}-a_{2}^{\dagger} a_{2}\right)
\end{aligned}
$$

This commutator vanishes whenever

$$
\Delta \zeta_{J}-J_{12} \zeta_{\Delta}=J_{12} \zeta_{J}^{*}-J_{12}^{*} \zeta_{J}=0,
$$

namely

$$
\begin{aligned}
& \frac{\zeta_{\Delta}}{\Delta}=\frac{\zeta_{J}}{J_{12}}=\frac{\zeta_{J}^{*}}{J_{12}^{*}} \equiv K \quad \text { when } \Delta \neq 0, \\
& \frac{\zeta_{J}}{J_{12}}=\frac{\zeta_{J}^{*}}{J_{12}^{*}} \equiv K \text { and } \zeta_{\Delta}=0 \quad \text { when } \Delta=0
\end{aligned}
$$

where $K$ is a fixed real number. It should be emphasized that the conditions (33) apply for any environment, when $\mathrm{f}(\mathrm{t})$ is replaced by an operator acting on the environment (e.g. $c_{0}^{\dagger} c_{0}$ in the previous section), and are not restricted to the telegraph noise example discussed below.

Equation (33) requires that $\zeta_{J} / J_{12}$ should be a real number. Using the gauge choice (8) also for the $\zeta$ 's, this would require $\zeta_{u} / J_{u}=\zeta_{d} / J_{d}$, or $q_{\zeta}=q_{J}$, where

$$
q_{J} \equiv J_{u} / J_{d}, \quad q_{\zeta} \equiv \zeta_{u} / \zeta_{d} .
$$

Since both $J_{u}$ and $J_{d}$ can be tuned by gate voltages, this condition can be achieved experimentally. Alternatively, as mentioned in the Introduction, one can imagine conditions under which $\zeta_{\Delta}=0$, e.g. when the noise source is placed symmetrically with respect to the two dots. In that case $\Delta$ can be tuned to zero, and Eq. (32) may still hold.

In both of these cases, $\mathcal{H}_{\mathrm{q}}$ and $\mathcal{V}$ can be diagonalized simultaneously. The corresponding eigenvalues are then

$$
\epsilon_{ \pm}= \pm \Omega / 2, \quad \mathcal{V}_{ \pm \pm}= \pm \zeta_{\Omega} / 2= \pm \sqrt{\zeta_{\Delta}^{2} / 4+\left|\zeta_{J}\right|^{2}},
$$

see Eq. (26). Denoting the corresponding common eigenstates by $|+\rangle$ and $|-\rangle$ (do not confuse with the \pm states of the fluctuator), the equations of motion (24) in this new basis become

$$
\begin{aligned}
& (\mathbf{I} s-\mathbf{W}) \widetilde{\boldsymbol{\rho}}_{ \pm \pm}=\boldsymbol{\rho}_{ \pm \pm}(0), \\
& (\mathbf{I} s-\mathbf{W}) \widetilde{\boldsymbol{\rho}}_{+-}=\boldsymbol{\rho}_{+-}(0)-i \boldsymbol{\Omega} \widetilde{\boldsymbol{\rho}}_{+-},
\end{aligned}
$$

with $\boldsymbol{\Omega}=\Omega \mathbf{I}+\zeta_{\Omega} \boldsymbol{\sigma}_{z}$.

The solution for the diagonal matrix elements is

$$
\widetilde{\boldsymbol{\rho}}_{ \pm \pm}=[\mathbf{I} s-\mathbf{W}]^{-1} \boldsymbol{\rho}_{ \pm \pm}(0) \equiv \widetilde{\mathbf{P}} \boldsymbol{\rho}_{ \pm \pm}(0),
$$

where $\widetilde{\mathbf{P}}$ is the Laplace transform of $\mathbf{P}(t)$, and therefore

$$
\boldsymbol{\rho}_{ \pm \pm}(t)=\mathbf{P}(t) \boldsymbol{\rho}_{ \pm \pm}(0) \equiv \boldsymbol{\rho}_{ \pm \pm}(0)=\rho_{ \pm \pm}(0) \mathbf{p}_{0},
$$

where we have used the explicit expressions (20) and (25). The components of these vectors are $\rho_{ \pm \pm}(t, b)=$ 
$p_{b} \rho_{ \pm \pm}(0)$, and the factor $p_{b}$ represents the probability of finding the stochastic noise at the state $b$. Averaging over this noise [Eq. [14] ], we find that $\rho_{ \pm \pm}(t)=\rho_{ \pm \pm}(0)$. Thus, the diagonal elements of the reduced density matrix do not approach the fully-mixed limit (5), implying partial decoherence. Note that these matrix elements are linear combinations of the original density matrix elements, with coefficients which involve the mapping from $|1,2\rangle$ to $|+,-\rangle$. In fact, one can repeat the above procedure for any operator which commutes with both $\mathcal{H}_{\mathrm{q}}$ and $\mathcal{V}$, see Eq. (7).

The second equation (36) yields

$$
\widetilde{\boldsymbol{\rho}}_{ \pm \mp}=\widetilde{\mathbf{F}}_{ \pm} \boldsymbol{\rho}_{ \pm \mp}(0) \equiv \widetilde{\mathbf{F}}_{ \pm} \rho_{ \pm \mp}(0) \mathbf{p}_{0}
$$

where

$$
\begin{aligned}
& \widetilde{\mathbf{F}}_{ \pm} \equiv[s \mathbf{I}-\mathbf{W} \pm i \boldsymbol{\Omega}]^{-1} \\
& =\frac{(2 s \pm 2 i \Omega+\lambda) \mathbf{I}+\lambda\left[\boldsymbol{\sigma}_{x}+\Delta p\left(\boldsymbol{\sigma}_{z}+i \boldsymbol{\sigma}_{y}\right)\right] \mp 2 i \zeta_{\Omega} \boldsymbol{\sigma}_{z}}{2\left[(s \pm i \Omega)(s \pm i \Omega+\lambda) \pm i \lambda \zeta_{\Omega} \Delta p+\zeta_{\Omega}^{2}\right]}
\end{aligned}
$$

Here we have used Eqs. (18) and (19). Averaging over the noise [Eq. (14)], $\rho_{+-}$is obtained noting that the average of $\boldsymbol{\sigma}_{x} \mathbf{p}_{0}$ is equal to 1 , while the averages of $-i \boldsymbol{\sigma}_{y} \mathbf{p}_{0}$ and $\boldsymbol{\sigma}_{z} \mathbf{p}_{0}$ are equal to $\Delta p$. The inverse Laplace transform then yields

$$
\begin{aligned}
& \rho_{+-}(t)=\left(A_{+} e^{\alpha_{+} t}+A_{-} e^{\alpha_{-} t}\right) \rho_{+-}(0) \\
& A_{ \pm}=\frac{ \pm\left(\lambda-2 i \zeta_{\Omega} \Delta p\right)+\sqrt{\lambda^{2}-4 \zeta_{\Omega}^{2}-4 i \lambda \zeta_{\Omega} \Delta p}}{2 \sqrt{\lambda^{2}-4 \zeta_{\Omega}^{2}-4 i \lambda \zeta_{\Omega} \Delta p}}
\end{aligned}
$$

where

$$
2 \alpha_{ \pm}=-2 i \Omega-\lambda \pm \sqrt{\lambda^{2}-4 \zeta_{\Omega}^{2}-4 i \lambda \zeta_{\Omega} \Delta p} .
$$

Therefore, $\boldsymbol{\rho}_{+-}(t)$ oscillates and decays to zero. This asymptotic limit can also be obtained using Eq. (27). The real parts of $-\alpha_{ \pm}$represent two decay rates. For weak coupling between the qubit and the environment, $|\zeta| \ll \lambda$, the shorter time (associated with $\alpha_{-}$) is of order $1 / \lambda$, the typical time between the fluctuator jumps. To leading order in $\zeta / \lambda$, the longer relaxation time $\tau$ is approximately given by

$$
\tau^{-1}=-\operatorname{Re}\left[\alpha_{+}\right] \approx 4 \zeta_{\Omega}^{2} p_{+} p_{-} / \lambda \equiv \zeta_{\Omega}^{2} S(0) / 2
$$

[see Eq. (22)]. This relation with the zero-frequency noise spectrum coincides with the well-known 'dephasing' time $T_{2} \equiv 2 \tau$, generated by fluctuations of the offdiagonal coupling between the two energy states, $, 14,23$ Equation (42) is also the same as that found (using a different method) by Itakura and Tokura, 12 for the special case when $\Delta=\zeta_{\Delta}=\operatorname{Im}\left[J_{12}\right]=\operatorname{Im}\left[\zeta_{J}\right]=0$.

In the NMR terminology one distinguishes between purely dephasing noise, which causes the decay of the off-diagonal element of the reduced density matrix (in the relevant basis), and purely relaxaional noise, associated with the decay of the diagonal matrix elements towards $1 / 2$. These two types of noise are associated with the decay times $T_{2}$ and $T_{1}$, respectively $\stackrel{4,5}{2}$ The model discussed in this section describes a purely dephasing mechanism, namely $T_{1} \rightarrow \infty$. Similar models have been treated in connection with a coupling to a continuum of phonon modes $\stackrel{24}{2}$ The result (43) is the same as that obtained for white noise, when $S(\omega)$ is independent of $\omega$. This result corresponds to the 'motional narrowing limit' in NMR, when the rate of the noise fluctuation $\lambda$ is the shortest time in the problem $\underline{\underline{5}}$

In the basis of the Hamiltonian eigenstates $| \pm\rangle$, the off-diagonal element $\rho_{+-}$decays to zero, and its magnitude has been used to quantify decoherence $\underline{\underline{12}}$ However, this decay is basis-dependent: in the original basis of the qubit dot states the off-diagonal matrix elements approach non-zero values. This is true whenever one encounters partial decoherence. Therefore, using offdiagonal elements to characterize decoherence may be misleading. It is better to quantify decoherence via a basis-independent measure, e.g. $\left[1-\operatorname{Tr} \rho^{2}\right] \equiv 2\left(\rho_{11} \rho_{22}-\right.$ $\left.\left|\rho_{12}\right|^{2}\right)$. This quantity approaches $1 / 2$ for the fully-mixed state, but is larger than $1 / 2$ for partial decoherence.

\section{B. Symmetric case}

Equation (33) refers to two cases: either the Hamiltonian contains an energy gap $\Delta$, and an associated noise parameter $\zeta_{\Delta}$, or both of these variables vanish. Since the results are qualitatively the same in both cases, we present explicit expressions for the simpler case $\Delta=\zeta_{\Delta}=0$. In this case, $\Omega=2\left|J_{12}\right|, \zeta_{\Omega}=2\left|\zeta_{J}\right|$, and the two common eigenstates are easily identified as

$$
| \pm\rangle=\left(|1\rangle \mp e^{-i \theta}|2\rangle\right) / \sqrt{2},
$$

where $\theta$ is defined in Eq. (8) (and simultaneously $\zeta_{J}=$

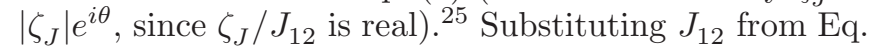
(8) then yields

$$
\tan \theta=\frac{\sin \phi}{q_{J}+\cos \phi}
$$

with $q_{J}=q_{\zeta}$ given in Eq. (34).

We can now use the results from the previous subsection. For that, we need to map the reduced density matrix from our original basis $\{1,2\}$ to the 'bondingantibonding' basis (44) and back. Substituting the initial conditions

$$
\begin{aligned}
& Z(0) \equiv \rho_{++}(0)-\rho_{--}(0)=2 \operatorname{Re}\left[e^{-i \theta} \rho_{12}(0)\right], \\
& \rho_{+-}(0)=\left[\rho_{11}(0)-\rho_{22}(0)\right] / 2+i \operatorname{Im}\left[e^{-i \theta} \rho_{12}(0)\right]
\end{aligned}
$$

into Eqs. (38) and (41) yields $\rho_{ \pm \pm}(t)$ and $\rho_{+-}(t)$, and the relations

$$
\begin{aligned}
& z(t) \equiv \rho_{11}(t)-\rho_{22}(t)=2 \operatorname{Re}\left[\rho_{+-}(t)\right] \\
& \rho_{12}(t)=e^{i \theta}\left(i \operatorname{Im}\left[\rho_{+-}(t)\right]-\left[\rho_{++}(t)-\rho_{--}(t)\right] / 2\right)
\end{aligned}
$$


yield the reduced density matrix in the original basis. It is now easy to check that at long times the diagonal elements $\rho_{11}$ and $\rho_{22}$ approach $1 / 2$, but the off-diagonal element approaches $\rho_{12}(t \rightarrow \infty) \rightarrow e^{i \theta} \operatorname{Re}\left[e^{-i \theta} \rho_{12}(0)\right]$, as in Eq. (91).

To present our results graphically, we follow conventional notations ${ }^{6}$ and write the reduced density matrix in the form

$$
\rho \equiv(\mathbf{I}+\mathbf{r} \cdot \boldsymbol{\sigma}) / 2,
$$

where the (real) Bloch vector $\mathbf{r} \equiv(x, y, z)$ is defined by
The full thick lines in Fig. 2 show the time evolution of the average components of the Bloch vector in the symmetric case, for one example of the parameters. Indeed, both the real and the imaginary parts of $\rho_{12}$ approach finite limits, while $z \rightarrow 0$. These limits, given by Eq. (9), are shown by thin lines.

$$
\rho_{11} \equiv(1+z) / 2, \quad \rho_{22} \equiv(1-z) / 2, \quad \rho_{12} \equiv(x-i y) / 2 .
$$
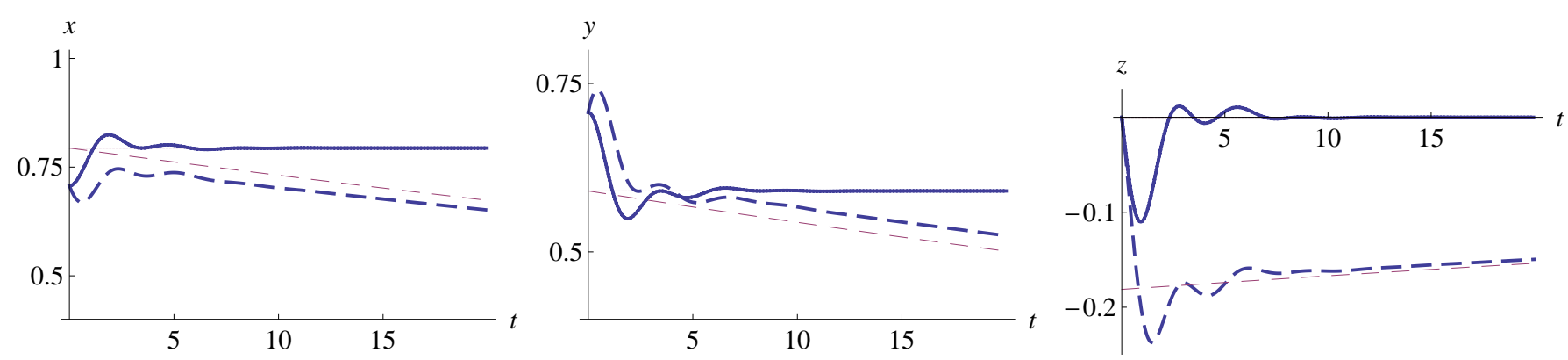

FIG. 2: The averages of $x, y$ and $z$ [Eq. [49]] for $\phi=-.3 \pi, J_{d}=.5, \zeta_{d}=.3, q_{J}=q_{\zeta}=.5, \zeta_{\Delta}=0, p_{+}=p_{-}=1 / 2$. The initial qubit state [Eq. (1)] is given by $\alpha=\gamma=.25 \pi$. All energies and inverse times are in units of $\lambda$. The full (dashed) thick lines correspond to $\Delta=0(0.2)$. The full (dashed) thin lines represent the exact (approximate) asymptotic behavior. The derivation of the dashed lines, for $\Delta \neq 0$, is described in Sec. V.

We now present more details on the derivation of Eq. (13). The general expression for the circulating current is given in Eq. (12). With the noise, this equation gives the conditional averages, so that we need to average over $\mathbf{J}_{u} \boldsymbol{\rho}_{21}$. In the stationary state one has $\boldsymbol{\rho}_{12} \rightarrow \rho_{12}(\infty) \mathbf{p}_{0}=e^{i \theta} \operatorname{Re}\left[e^{-i \theta} \rho_{12}(0)\right] \mathbf{p}_{0}$ [see Eq. (9)] ]. Substituting also $\mathbf{J}_{u}=J_{u} \mathbf{I}+\zeta_{u} \boldsymbol{\sigma}_{z}$, and noting that the average of $\boldsymbol{\sigma}_{z} \mathbf{p}_{0}$ is equal to $\Delta p$ and that $J_{u}$ and $\zeta_{u}$ are real, one obtains Eq. (13). Figure 3 shows the fluxdependence of this asymptotic current, for $q_{J}=q_{\zeta}=1 / 2$ and several values of the initial qubit relative phase $\gamma$ [Eq. (11)]. Interestingly, the current is odd (even) in $\phi$ for $\gamma=0$ $(\gamma=\pi / 2)$, but is neither odd nor even for intermediate values of $\gamma$. Equation (13) gives the current in terms of the initial qubit parameters $\alpha$ and $\gamma$. This current can in principle be measured by measuring the orbital magnetic moment of the electron on the ring. To retrieve $\alpha$ and $\gamma$, we need to perform three preliminary measurements. Fixing the flux at a non-trivial value $\phi_{1}$ (not an integer multiple of $\pi$ ), one should measure the asymptotic current for two known initial states. These measurements determine the device parameters $\theta_{1}$ and $J_{0}$. Repeating the same procedure for another flux $\phi_{2}$ and one known initial state, one would find $\theta_{2}$. The information on an unknown initial state can then be extracted by measuring the asymptotic current for the same two fluxes.

\section{GENERAL CASE}

Since the calculations for the general case are somewhat technical, we start with a brief summary of the results. Below we present the full solution for the time evolution of the reduced density matrix, which we have used to plot the dashed thick lines in Fig. 2. In this figure, as well as in much of the discussion below, we concentrate on small deviations from the symmetric case, namely

$$
\left|\Delta \zeta_{J}-J_{12} \zeta_{\Delta}\right| \ll \lambda, \Omega, \quad\left|q_{J}-q_{\zeta}\right| \ll 1
$$

see Eqs. (32)-(34). As can be seen from the figure, all three components of the Bloch vector [Eq. [49)] exhibit transient oscillations and then decay with a simple exponential,

$$
x \approx x_{0} e^{-t / \tau_{0}}, \quad y \approx y_{0} e^{-t / \tau_{0}}, \quad z \approx z_{0} e^{-t / \tau_{0}} .
$$




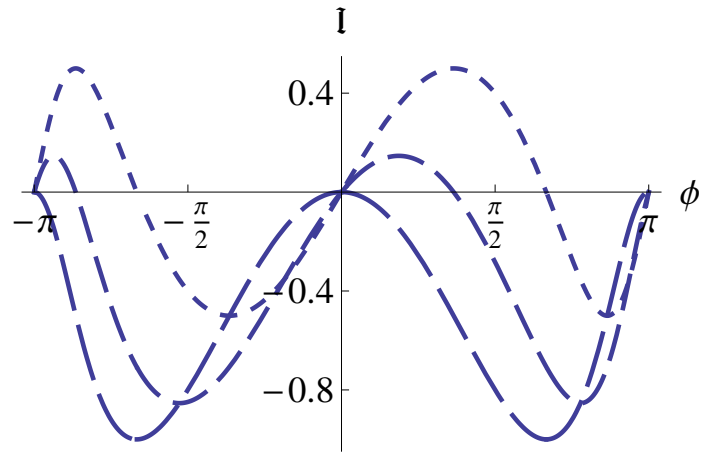

FIG. 3: The flux dependence of the asymptotic average current (in units of $J_{0}$ ) in the symmetric case, for $q_{J}=q_{\zeta}=1 / 2$ and $\alpha=.25 \pi$. Increasing dashes correspond to $\gamma=0, \pi / 4$ and $\pi / 2$.

To leading order in the small parameters (50), the slow decay rate is found to be

$$
\tau_{0}^{-1} \approx \frac{\left(\zeta_{\Omega} \Delta-\zeta_{\Delta} \Omega\right)^{2}+4 J_{\mathcal{I}}^{2}\left(\zeta_{\Delta}^{2}+\zeta_{\Omega}^{2}\right)}{\lambda\left[\lambda^{2}\left(\Omega+\zeta_{\Omega} \Delta p\right)^{2}+\left(\Omega^{2}-\zeta_{\Omega}^{2}\right)^{2}\right]},
$$

where

$$
\begin{aligned}
& \Omega=\Delta_{+-}=2 \operatorname{Re}\left[J_{12}\left|\zeta_{J}\right| / \zeta_{J}\right], \\
& \zeta_{\Omega} \equiv 2\left|\zeta_{J}\right|, \quad J_{\mathcal{I}} \equiv\left(q_{\zeta}-q_{J}\right) \sin \theta .
\end{aligned}
$$

Expanding also in $\zeta_{\Omega}$, this rate becomes

$$
\tau_{0}^{-1} \approx \frac{\left(\zeta_{\Omega} \Delta-\zeta_{\Delta} \Omega\right)^{2}+4 J_{\mathcal{I}}^{2}\left(\zeta_{\Delta}^{2}+\zeta_{\Omega}^{2}\right)}{2 \Omega^{2}} S(\Omega)
$$

[see Eq. (22)]. Again, the relaxation time is related to the spectral function of the noise. The corresponding decay time, $\tau_{0}$, indeed becomes infinite in the symmetric limit $\zeta_{\Omega} \Delta / \Omega-\zeta_{\Delta}=J_{\mathcal{I}}=0$, and remains very long for small symmetry breaking. This explains the behavior observed in Fig. 2. Unlike Eq. (43), the power spectrum function $S$ now picks the Rabi frequency $\Omega$ of the system, representing what Abragam calls the 'adiabatic modulation' $\underline{\underline{5}}$ This emphasis on the Rabi frequency is sometimes also called the 'rotating wave approximation'. The long relaxation time $\tau_{0}$ can be identified with the relaxational time $T_{1}$, responsible for the asymptotic decay of the diagonal elements of the density matrix towards equal occupations (associated with the decay of $Z$ [Eq. (46)], which did not decay in the symmetric case).

Below we also evaluate the amplitudes $x_{0}, y_{0}$ and $z_{0}$ [Eq. (51)], and the approximate results are shown by the thin dashed lines in Fig. 2. The slow exponential decay gives an opportunity to measure these amplitudes even after a long time. Unlike the symmetric case, where asymptotically $z \rightarrow 0$, one now finds non-zero values for the occupation difference $z=\rho_{11}-\rho_{22}$. The flux dependence of the amplitude $z_{0}$ is shown in Fig. 4. As in Fig. 3. note the even-odd dependence of $z(\phi)$. However, all these coefficients can be tuned by a few preliminary

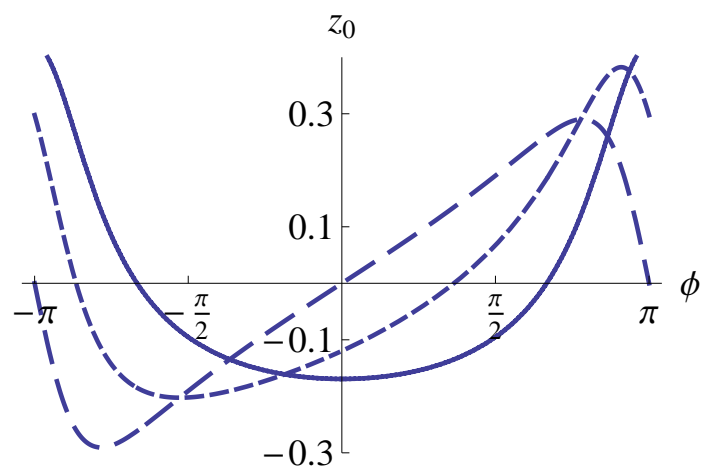

FIG. 4: The flux dependence of the amplitude $z_{0}$ for the same parameters as for the dashed lines in Fig. 2 but with $\gamma$ as in Fig. 3

experiments done for a few initial qubit states and a few fluxes, as discussed in connection with Fig. 3 ,

We now give more details. The unmotivated reader is welcome to move to the next section. Since we mainly consider small deviations from the symmetric case discussed in the previous section, we choose to stay with the same basis used for that case, namely Eq. (44). However, in order to decrease the number of noise-related terms, we choose the phase $\theta$ as the phase of $\zeta_{I}$, so that $\zeta_{J} e^{-i \theta}=\left|\zeta_{J}\right|$ becomes real. This requirement is equivalent to Eq. (45), upon replacing $q_{J}$ with $q_{\zeta}$ (in the previous section we had $q_{J}=q_{\zeta}$ ). With the basis (44), the two Hamiltonian operators become

$$
\begin{aligned}
& \mathcal{H}_{\mathrm{q}}=(\Omega / 2)(|+\rangle\langle+|-|-\rangle\langle-|)-\left(J_{+-}|+\rangle\langle-|+\text {h.c. }\right), \\
& \mathcal{V}=\left(\zeta_{\Omega} / 2\right)(|+\rangle\langle+|-|-\rangle\langle-|)+\left(\zeta_{\Delta} / 2\right)(|+\rangle\langle-|+\text { h.c. }), \\
& J_{+-}=-\Delta / 2+i J_{\mathcal{I}}
\end{aligned}
$$

[see also Eq. (53)]. As a result, Eqs. (36) are now generalized to the form

$$
\begin{aligned}
& (s \mathbf{I}-\mathbf{W}) \widetilde{\mathbf{Z}}=\mathbf{Z}(0)+2 i\left(\mathbf{J}_{+-} \widetilde{\boldsymbol{\rho}}_{-+}-\mathbf{J}_{-+} \widetilde{\boldsymbol{\rho}}_{+-}\right), \\
& (s \mathbf{I}-\mathbf{W} \pm i \boldsymbol{\Omega}) \widetilde{\boldsymbol{\rho}}_{ \pm \mp}=\boldsymbol{\rho}_{ \pm \mp}(0) \mp i \mathbf{J}_{ \pm \mp} \widetilde{\mathbf{Z}} .
\end{aligned}
$$

Noting that

$$
\widetilde{\mathbf{P}} \mathbf{p}_{0}=\frac{1}{s+\lambda}\left(1+\frac{\lambda}{s} \mathbf{T}\right) \mathbf{p}_{0}=\frac{1}{s} \mathbf{p}_{0}
$$

the first equation yields

$$
\widetilde{\mathbf{Z}}=\mathbf{Z}(0) / s+2 i \widetilde{\mathbf{P}}\left(\mathbf{J}_{+-} \widetilde{\boldsymbol{\rho}}_{-+}-\mathbf{J}_{-+} \widetilde{\boldsymbol{\rho}}_{+-}\right) .
$$

Substituting this equation into the equations for $\widetilde{\boldsymbol{\rho}}_{ \pm \mp}$, yields two coupled equations for the latter two 2component vectors.

Solving these equations, and performing the inverse Laplace transform, one finds that the time dependence of the off-diagonal element of the density matrix $\boldsymbol{\rho}_{+-}(t)$ is a sum over exponential terms, $e^{\alpha_{\ell} t}$, where the $\alpha_{\ell}$ 's are 
poles of $\widetilde{\boldsymbol{\rho}}_{+-}(s)$, found as the roots of a sixth order real polynomial,

$$
d(s)=\sum_{\ell=0}^{6} d_{\ell} s^{\ell} .
$$

Substituting the solutions for $\widetilde{\boldsymbol{\rho}}_{ \pm \mp}$ into Eq. (58) yields $Z(t)$. Equations (47) are then used to derive the time dependence of the average Bloch vector $(x, y, z)$, as shown by the dashed lines in Fig. 2. Interestingly, there seem to be two main time scales. At the beginning one observes a transient oscillatory behavior, up to time scales of the order given by the symmetric case, Eq. (42). After that, all three variables exhibit a very slow pure exponential decay, see Eq. (51).

To explain this asymptotic behavior, we return to the polynomial (59). The long-time limit of the solutions is determined by the behavior of $d(s)$ at small Laplace variable $s$, where we can use the approximation $d(s) \approx$ $d_{0}+d_{1} s$. The Laplace transform then decays as $e^{\alpha_{0} t}=$ $e^{-t / \tau_{0}}$, where $\tau_{0}^{-1}=-\alpha_{0} \approx d_{0} / d_{1}$. This approximation is valid as long as $d_{0} / d_{1}$ is small. We find

$$
d_{0}=4 \lambda^{2} p_{+} p_{-}\left[\left(\zeta_{\Omega} \Delta-\zeta_{\Delta} \Omega\right)^{2}+4 J_{\mathcal{I}}^{2}\left(\zeta_{\Delta}^{2}+\zeta_{\Omega}^{2}\right)\right] .
$$

Therefore, $\tau_{0}$ becomes infinite, and one has a non-trivial stationary solution, only when $d_{0}=0$, which happens only when $J_{\mathcal{I}}=0$ and either $\Delta=\zeta_{\Delta}=0$ or $\zeta_{\Delta} / \Delta=$ $\zeta_{\Omega} / \Omega=\left|\zeta_{J}\right| / \operatorname{Re}\left[J_{12} e^{-i \theta}\right]$, consistent with the results in Sec. IV. In all other cases, $\widetilde{\boldsymbol{\rho}}_{+-}$and $\widetilde{\mathbf{Z}}$ approach finite limits as $s \rightarrow 0$, and therefore Eq. (27) implies that all the components of the Bloch vector approach zero as $t \rightarrow \infty$, leading to the fully-mixed limit (5).

When the deviation from the symmetric case is small, it is appropriate to expand the results in powers of $\left(\zeta_{\Omega} \Delta / \Omega-\zeta_{\Delta}\right)$ and $J_{\mathcal{I}} / \Omega$. In our case, neglecting higherorder terms in $\Delta, \zeta_{\Delta}$ and $J_{\mathcal{I}}$, one has $d_{1} \approx \lambda\left[\lambda^{2}(\Omega+\right.$ $\left.\left.\zeta_{\Omega} \Delta p\right)^{2}+\left(\Omega^{2}-\zeta_{\Omega}^{2}\right)^{2}\right]$, yielding Eq. (52).

The coefficients $x_{0}, y_{0}$ and $z_{0}$ in Eq. (51) are the residues of the poles of the corresponding Laplace transforms at $s=\alpha_{0}=-\tau_{0}^{-1}$. Therefore, to leading order in $\mathbf{J}_{ \pm \mp}$, these residues are the same as those for the pole at $s=0$ in the symmetric case. To this leading order,

$$
x_{0}-i y_{0}=e^{-i \theta} \sin (2 \alpha) \cos (\theta+\gamma)
$$

and $z_{0}=0$ [see Eq. (9)]. Consequently, the amplitude for the slow exponential decay of the circulating current is also approximately given by Eq. (13), as plotted in Fig. 3. Measuring this amplitude therefore gives the same information as discussed in connection with that equation (see the end of Sec. IV.B).

Corrections to the next order in $\mathbf{J}_{ \pm \mp}$ just shift the values of $x_{0}$ and $y_{0}$ slightly, and therefore we do not discuss them here. In contrast, these corrections are crucial for $z_{0}=2 \operatorname{Re}\left[\rho_{+-}\right]\left[\right.$Eq. [47)], since $z_{0}=0$ at the zeroth order. To first order in $\mathbf{J}_{+-}=-\left(\Delta \mathbf{I}+\zeta_{\Delta} \boldsymbol{\sigma}_{z}\right) / 2+i J_{\mathcal{I}} \mathbf{I}$, the second Eq. (56) becomes

$$
\widetilde{\boldsymbol{\rho}}_{+-}=\widetilde{\mathbf{F}}_{+}\left[\boldsymbol{\rho}_{+-}(0)-i \mathbf{J}_{+-} \mathbf{Z}(0) / s\right] .
$$

The amplitude $z_{0}$ is given by the average of $2 \operatorname{Re}\left[\lim _{s \rightarrow 0}\left(s \widetilde{\boldsymbol{\rho}}_{+-}\right)\right]$. Some algebra then yields

$$
\begin{aligned}
& z_{0} \approx \lambda\left(c_{1} \zeta_{\Delta}+c_{2} \Delta+c_{3} J_{\mathcal{I}}\right) Z(0) / d_{1}, \\
& c_{1}=\lambda^{2}\left(\Omega+\zeta_{\Omega} \Delta p\right) \Delta p+\left(\Omega^{2}-\zeta_{\Omega}^{2}\right)\left(\Omega \Delta p-\zeta_{\Omega}\right), \\
& c_{2}=\lambda^{2}\left(\Omega+\zeta_{\Omega} \Delta p\right)+\left(\Omega^{2}-\zeta_{\Omega}^{2}\right)\left(\Omega-\zeta_{\Omega} \Delta p\right), \\
& c_{3}=8 \lambda p_{+} p_{-} \zeta_{\Omega}^{2},
\end{aligned}
$$

where $Z(0)=\sin (2 \alpha) \cos (\theta+\gamma)$. The thin dashed lines in Fig. 22 were drawn using Eqs. (51), (61) and (63). As can be seen, the approximation for $z$ is excellent, while those for $x$ and $y$ are good apart from a small shift which can be calculated from the next order. The $\phi$-dependence of $z_{0}$ is quite complicated, since the coefficients $c_{\ell}$ also depend on $\phi$, via $\Omega$ and $\zeta_{\Omega}$. When $q_{J} \neq q_{\zeta}$ then $J_{\mathcal{I}}$ is proportional to $\sin \theta$, introducing an additional $\phi$-dependence.

\section{DISCUSSION}

In this paper we discussed a qubit which is coupled to the environment via a single telegraph noise variable $f(t)$. Apart from the quantum information, which is stored in the qubit, the system is characterized by the following parameters: the bare energy gap $\Delta$, the bare hopping energies $J_{d}$ and $J_{u}=J_{d} q_{J}$, and the amplitudes of the noise $\zeta_{\Delta}, \zeta_{d}$ and $\zeta_{u}=\zeta_{d} q_{\zeta}$. To obtain partial decoherence we require $\Delta=\zeta_{\Delta}=0$ or $\zeta_{\Delta} / \Delta=\zeta / J_{0}$ and $q_{J}=q_{\zeta}$. As already mentioned, the energy gap $\Delta$ and the two hopping energies $J_{u}$ and $J_{d}$ can all be tuned by gate voltages on the two quantum dots and on the barriers along the hopping paths. Therefore, one can in principle tune these parameters to the partially decoherent limit.

Furthermore, $\zeta_{\Delta}$ depends on the relative locations of the noise source and the qubit. To reduce $\zeta_{\Delta}$, the noise source should be placed symmetrically relative to the two dots. In that case, $\zeta_{1}=\zeta_{2}$, and therefore $\zeta_{\Delta}=0$. Also, if $\epsilon_{1}$ and $\epsilon_{2}$ represent two arbitrary levels of some large dot, they will not be strongly affected if the volume of that dot is not sensitive to the noise. For an arbitrary qubit state and an arbitrary flux, we thus propose to tune $\Delta$ and $q_{J}$ until one observes a non-zero asymptotic circulating current, which also generates an orbital magnetic moment. After such tuning one can use the same system for retrieving the quantum information, stored initially on the qubit, from measuring the current and/or the magnetic moment for any other flux and any other initial qubit state.

The measurement of equilibrium persistent currents is quite difficult, and it is only recently that novel methods were invented to measure them $\underline{26}$ It remains to be seen if such methods can also be applied to the circulating currents discussed in the present paper. As mentioned, when the system's parameters deviate from the special cases with partial decoherence then one can also extract the initial qubit information from measurements of the occupations of the states on the qubit's quantum dots. 
Measuring the qubit dots occupations at real time is difficult, since the measuring time should be much shorter than any decoherence time $\stackrel{27}{2}$ However, in the scheme presented here one need not worry about the fast transient decay times, and the long decay time $\tau_{0}$ can in fact be tuned experimentally. All one needs to do is tune the necessary gate voltages and watch for a slow relaxation. Therefore, there is a much better chance that existing methods for measuring dot occupations will work here.

Equation (7) suggests other options for 'symmetric' cases. For example, if $J_{12}=\zeta_{J}=0$ then $z=\rho_{11}-\rho_{22}$ remains constant in time, while $\rho_{12}$ decays to zero. In this case, measuring the time-independent dot occupations will yield $z(0)=\cos (2 \alpha)$ [Eq. (11)]. However, to gain the flexibility due to the magnetic flux, and to extract information on $\gamma$, one would still need to deviate slightly from this symmetric case. An expansion in $J_{12}$ and/or in $\zeta_{J}$ would then yield similar slow decays towards the fully-mixed state.
We expect similar qualitative results for more complex structures. For example, one can replace each of the bonds $u$ and $d$ by a path which goes via a linear chain of quantum dots, and one can tune the energy level on one or more of these dots through a resonance, thus changing the effective hopping energy $J_{u, d}$. As stated, we also expect similar results for other sources of noise and for a system affected by more than one fluctuator, provided one can tune the system to a 'symmetric' limit where the commutator $\left[\mathcal{H}_{\mathrm{q}}, \mathcal{V}\right]$ is small.

\section{Acknowledgements}

We acknowledge discussions with Y. Imry. SD is grateful to BGU and to the Einstein center at the WIS for partially supporting his visits to Israel. AA and OEW acknowledge support from the DIP and from the BSF, as well as discussions with P. Stamp and the hospitality of the PITP at UBC.
* Electronic address: aaharony@bgu.ac.il Also at Tel Aviv University, Tel Aviv 69978, Israel.

† Also at Tel Aviv University, Tel Aviv 69978, Israel.

1 C. Bennett, Phys. Today 48, 24 (1995); D. P. DiVincenzo, Science 270, 255 (1995); A. M. Steane, Rep. Prog. Phys. 61, 117 (1998)

2 D. Loss and D. P. DiVincenzo, Phys. Rev. A 57, 120 (1998); T. Tanamoto, Phys. Rev. A 61, 022305 (2000).

3 J. Q. You, X. Hu and F. Nori, Phys. Rev. B 72, 144529 (2005).

${ }^{4}$ C. P. Slichter, Principles of Magnetic Resonance (Harper and Row, New York, 1963).

5 A. Abragam, Principles of Nuclear Magnetism (Oxford University Press, New York 1961).

${ }^{6}$ L. Allen and J. H. Eberly, Optical Resonance and TwoLevel Atoms (Dover publivations, New York, 1987).

7 Y. Nakamura, Yu. A. Pashkin, and J. S. Tsai, Nature (London) 398, 786 (1999).

8 W. H. Zurek, Phys. Today 44 (no. 10), 36 (1991).

9 D. A. Lidar, I. L. Chuang, and K. B. Whaley, Phys. Rev. Lett. 81, 2594 (1998).

10 P. Zanardi and M. Raseti, Phys. Rev. Lett. 79, 3306 (1998).

11 See for instance S. Dattagupta, Relaxation Phenomena in Condensed Matter Physics (Academic Press Orlando, 1987).

12 T. Itakura and Y. Tokura, Phys. Rev. B 67, 195320 (2003).

13 e.g. J. Bergli, Y. M. Galperin, and B. L. Altshuler, New J. Phys. 11, 025002 (2009); B. Cheng, Q. H. Wang, and R. Joynt, Phys. Rev. A 78, 022313 (2008) and references therein.

14 S. A. Gurvitz and D. Mozyrsky, Phys. Rev. B 77, 075325 (2008).

15 See also S. A. Gurvitz, D. Mozyrsky, and G. P. Berman, Phys. Rev. B 72, 205341 (2005); S. A. Gurvitz and Ya. S. Prager, Phys. Rev. B 53, 15932 (1996); S. A. Gurvitz, Phys. Rev. B 57, 6602 (1998).
16 For other approaches, see e.g. B. Abel and F. Marquardt, Phys. Rev. B 78, 201302 (2008); I. V. Yurkevich, J. Balwin, I. V. Lerner, and B. L. Altshuler, Phys. Rev. B 81, 121305 (2010).

17 However, coupling the phase of the hopping amplitudes to a spin bath generates a slower decay, as a power of the time; see e.g. Z. Zhu, A. Aharony, O. Entin-Wohlman, and P. C. E. Stamp, Phys. Rev. A 81, 062127 (2010); D. Sokolovski and S. A. Gurvitz, Phys. Rev. A 79, 032106 (2009).

18 Y. Imry, Introduction to Mesoscopic Physics, 2nd ed. (Oxford University Press, Oxford, 2002).

19 R. Kubo, in Fluctuation, Relaxation and Resonance in Magnetic systems (D. ter Haar, editor), (Oliver and Boyd, Edinburg, 1962); also in Adv. Chem. Phys. 15, 101 (1969).

20 M. Blume, Phys. Rev. 174, 351 (1968).

21 See Chapter VIII of Ref. 11 .

22 Y. M. Galperin, B. L. Altshuler and D. V. Shantev, in Fundamental Problems of Mesoscopic Physics, ed I. V. Lerner et al. (Dordrecht: Kluwer), pp. 14165.

23 A.J. Leggett, S. Chakravarty, A.T. Dorsey, M. P. A. Fisher, A. Garg, and W. Zwerger, Rev. Mod. Phys. 59, 1 (1987).

24 See e.g. L. Viola and S. Lloyd, Phys. Rev. A 58, 2733 (1998).

25 An alternative way to solve the problem for a complex $J_{12}$ is to perform a gauge transformation from $|2\rangle$ to $\left|2^{\prime}\right\rangle=$ $e^{-i \theta}|2\rangle$. Then $J_{12}$ becomes real, the Hamiltonian becomes symmetric under $1 \leftrightarrow 2^{\prime}$ and one can use the same solution as in Ref. 12. That is why we call this solution 'symmetric'.

26 See e.g. H. Bluhm, H., N. Koshnick, J. Bert,M. Huber, and K. Moler, Phys. Rev. Lett. 102, 136802 (2009); A. C. Bleszynski-Jayich et al., Science 326, 272 (2009) and references therein.

27 Many methods to measure dot occupation are reviewed by T. Fujisawa, T. Hayashi and S. Sasaki, Rep. Prog. Phys. 69, 759 (2006). 